\title{
Correction to: Nuclear Fusion
}

\author{
Edward Morse
}

\section{Correction to:}

E. Morse, Nuclear Fusion, Graduate Texts in Physics, https://doi.org/10.1007/978-3-319-98171-0

The published version of this book included errors in Code listings, Figure replacement and Typo errors throughout the book. These rendering have been corrected and updated in the book. 